\title{
Giving a Talk
}

Bryna Kra

No one likes to sit through a bad talk, but unfortunately everyone does it much too frequently. No one sets out to give a bad talk, but probably all of us have done so. Paul Halmos [1] wrote a beautiful article on how to give a talk, and his advice remains apt today (and some of it is repeated here). But new technology, varying audiences, and different venues force modifications in how we give talks. This is subjective advice on how to give a good talk and, especially, how to avoid giving a bad talk. Of course, not all of it applies to everyone or to every situation, but my hope is that anyone reading this article is provoked into thinking a bit more when preparing a talk.

\section{What Kind of Talk?}

Think about the purpose of the talk: Is it a seminar? Colloquium? Conference? Job talk? What is the target audience: is it a survey talk? Is it aimed at graduate students? Is it meant for experts? The answers affect all aspects of the talk, starting with the format: an "old fashioned" chalk talk? Projecting via a document camera or overhead? A computer presentation?

There are no right or wrong answers, but before making a decision, think about the audience and the venue. For a survey talk with many references, a computer presentation might be appropriate. For a large conference, boards might not be available. For a small seminar, one can include details of a proof that can only be done on a board (or perhaps more accurately, should only be done on a board). Find out if the room is appropriate for using the board, particularly if it is a large room. When using technology, find out if the screen is easily visible, if it can be combined with use of a board, and if a computer is provided. These decisions depend on the culture of the venue for the talk, but with proper planning, any presentation method

Bryna Kra is professor of mathematics at Northwestern University. Her email address is kra@math. northwestern. edu.

The author was partially supported by NSF grant 1200971. DOI: http://dx.doi.org/10.1090/noti941 available can be used to give an excellent talk geared to the appropriate target audience.

\section{Plan and Scope}

The first piece of advice is obvious, but still needs stating: plan the talk. The talk shoud be a cohesive narrative, telling a story from the beginning to the end. Overall, plan to keep it simple. The whole audience should follow at least half of the talk, and most of the audience should be able to follow most of the talk. Do not just address the experts; save any comments meant for them until the last few minutes of the talk.

How does one go about planning? Perhaps the best advice is a negative piece of advice: do not try to cover too much! This especially applies to computer talks-just because it is easy to flash many theorems and explanations on a screen does not mean that anyone can follow. Instead, pick a focus. Do not set out to tell the audience every theorem in a subject or every theorem from a paper, but take the time to delve into a particular aspect.

Start with a hook: a general problem or subject likely to be of interest to the audience. By providing background, history, and placing the subject in a greater context, one can pique the audience's interest before delving into any details. The exact type of motivation depends, as always, on the purpose of the talk and the target audience. For example, a colloquium on patterns in the primes might start with the Twin Prime Conjecture and one on the wave equation might start with a picture or graphic, whereas for a talk in a number theory or PDE seminar, one should assume familiarity with these topics.

Take time in giving the definitions or stating a theorem, and make sure that the audience has the time to digest it. If at all possible, use examples or pictures. Speakers, especially young speakers, believe that the audience knows the subject better than they actually do. But even when this is true, introducing the basic definitions and statements is a time to set notation and to give the flavor of how the basics are used and how these notions fit into the topic. In spite of what many people believe, the reality is that the speaker has probably thought more about the topic than anyone else in the room, 
and even experts do not always remember all the definitions and basic theorems. Of course, one can go too far by including too many basics for the particular audience: for a talk in a dynamics seminar, there is no need to define the word ergodic, but one should in a colloquium.

A good rule of thumb (other than for expository talks) is that at least half the talk should be devoted to the speaker's own work. One should get to one's own work as early as is practical-the earlier the better. If left to the last five minutes, the audience does not have time to absorb the material and pose questions.

Everyone likes coming out of a talk having learned something, and the speaker should try to include some sort of proof, even in a general audience talk. It might be a very simple fact or a much simplified toy version of the real problem. If the talk is a seminar, it might be an outline of steps in a long proof or the proof of a crucial, technical lemma. If the statement or proof can be given in a simple case, do it. The audience does not need to hear the details of the most general setting in which the theorem can be proven and can instead be directed to the paper(s) concerning it. By avoiding the most general possible statement, much heavy (and incomprehensible) notation is also avoided. More important than the details is the motivation of the subject, an overview of the ideas, and the intuition behind them.

Talks are meant to convey something that is not in the literature and not just to be a repetition of the introduction of a recent article. A useful exercise is to think back to how the result was proven: what were false steps? What were examples used to test the ideas? Explaining such steps can be instructive components of a talk.

For a seminar or typical conference talk, one theorem or a few related theorems are usually enough material. If the theorems covered naturally split into two different papers, then this is probably material for two separate talks. This does not mean that any result from another article ought to be omitted, but rather it should be used to bolster the main focus of the talk. Surveys are different: they should go slowly and have a goal but likely include many more (interrelated) topics.

The last step of preparing a talk, particularly for inexperienced speakers, is practice. Practice the talk out loud and time it. Frequently, people are surprised to find that what they thought would be easy to fit into twenty minutes really takes forty-five minutes, or vice versa. Even experienced speakers benefit by saying all the words before the actual talk, even if each iteration is somewhat different. I still write every word that I plan to say, though I rarely refer to these extensive notes during the talk-just the exercise of thinking through the words improves the presentation.

\section{Citations and Thanks}

Make it clear which results are yours and which are in the literature. Give citations for any results used or previous results on the same topic; there is no need to give complete bibliographic listings, just the author(s) and year of publication suffice.

Always thank the host or hosts for the invitation. Remember to cite any coauthors and anyone else who helped with the work.

\section{Computer Talks}

The biggest change over the past ten years has been the proliferation of computer talks, leading to a host of self-created problems. The most basic problem is unreadable slides: the computer is not set up correctly for projection, the type is too small, or a slide is too crowded.

The first of these problems is easily corrected with a few minutes of planning. Check in advance that the file works in full screen mode and know how to put the computer into this mode. If at all possible, try the file on the computer to be used during the talk. Before the talk begins, make sure to close any other programs running in the background, especially anything that might interrupt the presentation with a pop-up.

The other two issues need to be addressed during the preparation of the talk. Simply put, computer talks do not work if the slides are too crowded. The type size has to be large, and it should be readable at the back of the room. This means that there is plenty of space between the lines and a maximum of about ten lines per slide (fewer is even better). Leave out as many words as possible and do not write full paragraphs. Beamer [2], a commonly used LATEX class for creating slides, as with most other presentation programs, comes with preset margins and spacings. They are designed so that one cannot put too much on a single slide, and there is no reason to override the settings.

There is also no reason to put every word on the slide and then do nothing more than read it aloud - if that is the plan, just post the slides on a website and forget about giving the talk. It is better to have less on the slides and then use many words to explain what it all means. You should be able to state everything on a slide in less than a minute, but spend at least two to three minutes per slide fleshing out the explanations. Many excellent speakers spend five to six minutes per slide.

A pointer or the mouse is useful for highlighting what the audience should focus on, but do not overdo it. Use it to highlight a particular object for a few seconds and then let the audience continue to look (or not) themselves. A bouncing laser pointer is a sure way to make the audience dizzy.

Some speakers like to reveal the lines of a presentation one line at a time. But this means that the last line is visible for only a few seconds before it 
is replaced by a new page. It is often better to show the whole slide at once and let the audience see what is coming. To draw the audience's attention to a particular statement, use the pointer, highlight the particular statement in another color (easy to do, for example, in Beamer), or use a lighter color to show the lower portions of the slide (again, easy in Beamer).

Slides are great for repeating something that is needed more than once. But that does not mean scrolling back to an earlier slide out of order. Instead, include another slide that repeats the result, instantly reminding the audience exactly what is needed-this is one of the benefits of a computer presentation.

Background graphics are almost always distracting. To get the audience to focus on the math, leave out the background of a beautiful sunset that obscures the equations and theorems. Some colors do not project well and should be avoided; particularly, many light colors do not work on rear projection screens. When in doubt, try the colors in advance on a real screen, not just on a small computer screen.

On the other hand, when all the talks look the same, the audience begins to glaze over. Judicious use of color on slides can break up the monotony without being distracting. Pictures and graphics can enhance a talk, and when appropriate, animation is an excellent tool for explaining an idea or a proof. The animations should be embedded in the file and tested in advance-no one enjoys watching a speaker search through hundreds of files on a computer.

\section{Short Talks}

Many conferences include short (twenty minutes or less) talks. Although one might think that these are easier to prepare, in some sense they are even harder. In twenty minutes, there is no time to present all the history, give a complete proof, or enter into the details of a construction. The goal is to convey a single result or several very closely related results, and this requires stripping out all other elements from the talk. Usually six to seven slides is more than enough for twenty minutes. Think of a twenty-minute talk as an extended movie trailer for the results: get the audience interested in finding out more.

Most advice for short presentations is the same as that for longer ones, just compressed: the results should be placed in context, important earlier results should be cited, and there should be a hint as to the ingredients that enter the proof. Heavy notation and long statements should be avoided, even if this means that the general statement is omitted or the statements are less precise (so long as the imprecisions are made clear to the audience).

\section{Language Issues}

For nonnative English speakers, giving a talk in English can often be a significant hurdle, but there are several ways to make it easier. The first is to practice the talk, even multiple times, with or without an audience. It may be easier to use a computer for the presentation and use the slides as a memory tool for difficult words or phrases. If it is more appropriate to give a chalk talk, a handout can help by summarizing key points. Always remember to speak in full sentences and try to explain everything as much as possible.

\section{Pet Peeves}

This is a personal list of egregious errors that I have witnessed. Having said that, I am sure that I have committed each of these violations at some point, but these errors can be easily avoided:

- Do not number your theorems. To refer back to a theorem, give it a memorable name, like the "Comparison Theorem" or the "Main Estimate". No one can remember what Theorem 2 was, much less Theorem 8!

- Do not use the same letter for two different purposes, even if this seems entirely natural. For example, if there is a map that is $C^{2}$ and a constant $c$, use $k$ for the constant. Instead of using both a capital and lowercase version of a letter, such as $\phi$ and $\Phi$, use different letters unless they are very closely linked. Otherwise, it is too hard to distinguish the two while talking, leading to awkward statements like "little $\phi$ " and "capital $\Phi$ ".

- Do not introduce new definitions or a new topic in the last five minutes (the exception being a short talk). Instead, use this time to sum up main points, describe future directions, or address the experts.

- Do not reveal your own insecurities with comments such as "my result is trivial" or "I would extend the result to case X, but I don't understand $X$ " or "I am sure everyone in the audience knows more about this." Never belittle your own work, as there are many other people who would be happy to do this for you.

\section{Finishing Up}

Do not rush the talk, but do not go over. It is better to finish with a minute or two left rather than keep impatient people in their seats for extra time. Be prepared for questions at the end and leave time for the audience to ask them.

Keep in mind that you, the speaker, know more about your work than the audience does. So relax, good luck, and enjoy yourself.

\section{References}

[1] Paul R. Halmos, How to talk mathematics, Notices Amer. Math. Soc. 21 (1974), 155-158.

[2] http://en.wikipedia.org/wiki/Beamer (LATEX). 